\title{
Cyclizations of $N$-Stannylaminyl Radicals onto Nitriles
}

\author{
Luisa Benati, ${ }^{\dagger}$ Giorgio Bencivenni, ${ }^{\dagger}$ Rino Leardini,$^{\dagger^{*}}$ Matteo Minozzi,,$^{\dagger}$ Daniele Nanni, ${ }^{\dagger}$ \\ Rosanna Scialpi, ${ }^{\dagger}$ Piero Spagnolo, ${ }^{r^{* *}}$ Giuseppe Zanardi ${ }^{\dagger}$ and Corrado Rizzoli \\ "Dipartimento di Chimica Organica "A. Mangini", Università di Bologna, \\ Viale Risorgimento 4, I-40136 Bologna, Italy. \\ "Dipartimento di Chimica Generale ed Inorganica, Analitica e Chimica Fisica, Università di Parma, \\ Viale delle Scienze 78, I-43100 Parma, Italy.
}

spagnolo@ms.fci.unibo.it

\section{SUPPORTING INFORMATION}

\section{Table of contents:}

Page S2. Experimental procedures and spectral data for new compounds.

Page S13. X-Ray diffraction details for compounds 10d and 11c.

Page S16. Beckwith-Houk transition state models for cyclization of aminoiminyl $\mathbf{1 2 d}\left(\mathrm{SnBu}_{3}=\mathrm{Me}\right)$.

Page S17. Optimized (DFT) structures of radicals cis-14b and trans-14b $\left(\mathrm{SnBu}_{3}=\mathrm{SnMe}_{3}\right)$, 3-ethyl-2imino-3-pyrrolidinecarbonitrile (3a), and tautomeric 5-amino-4-ethyl-3,4-dihydro-2Hpyrrole-4-carbonitrile.

Page S19. References. 
General Procedures. All melting points are uncorrected. ${ }^{1} \mathrm{H}$ and ${ }^{13} \mathrm{C}$ NMR spectra were recorded at 300 or $400 \mathrm{MHz}$ (75 or $100 \mathrm{MHz}$ for ${ }^{13} \mathrm{C}$ ) in deuteriochloroform, unless otherwise stated, using tetramethylsilane as the internal standard. Mass spectra were determined by the electron impact method (EI, $70 \mathrm{eV}$ ) or electron spray ionization (ESI). IR spectra were recorded in chloroform. Column chromatography was carried out on ICN silica gel (70-230 or 230-400 mesh) by gradual elution with mixtures of light petroleum (bp 40-70 ${ }^{\circ} \mathrm{C}$ ) and diethyl ether or dichloromethane or methanol and final elution with methanol. Previously reported reaction products were identified by spectral comparison. TBAB is tetrabutylammonium bromide. 2-Bromomethylbenzonitrile ${ }^{1}$ and 2-azidomethylbenzonitrile $(2)^{2}$ were prepared according to the literature.

Monoalkylated malononitriles. These compounds were synthesized in $70-98 \%$ yield from malononitrile, alkyl halide, TBAB, and potassium carbonate (or potassium tert-butoxide) according to the procedure of Diez-Barra. ${ }^{3}$ 2-Ethylmalononitrile, ${ }^{3}$ 2-benzylmalononitrile, ${ }^{3}$ 2-allylmalononitrile, ${ }^{3}$ 2$[(E)$-2-butenyl $]$-malononitrile, ${ }^{4} \quad$ 2-(3-methyl-2-butenyl)malononitrile, ${ }^{5} \quad$ and $\quad 2-[(E)-3$-phenyl-2propenyl]malononitrile ${ }^{6}$ are known compounds.

2-[(Z)-3-Hexenyl]malononitrile. Yield $=80 \%$; oil; IR $v_{\max }\left(\mathrm{cm}^{-1}\right) 2258(\mathrm{CN}) ;{ }^{1} \mathrm{H}$ NMR $(400 \mathrm{MHz}) \delta$ $0.99(3 \mathrm{H}, \mathrm{t}, J=7.6 \mathrm{~Hz}), 2.07-2.14(4 \mathrm{H}, \mathrm{m}), 2.39(2 \mathrm{H}, \mathrm{q}, J=7.6 \mathrm{~Hz}), 3.73(1 \mathrm{H}, \mathrm{t}, J=7.4 \mathrm{~Hz}), 5.19-$ $5.27(1 \mathrm{H}, \mathrm{m})$, 5.56-5.64 (1 H, m); ${ }^{13} \mathrm{C} \mathrm{NMR}(100 \mathrm{MHz}) \delta 14.79\left(\mathrm{CH}_{3}\right), 21.33\left(\mathrm{CH}_{2}\right), 22.24(\mathrm{CH}), 24.52$ $\left(\mathrm{CH}_{2}\right), 31.51\left(\mathrm{CH}_{2}\right), 113.24(\mathrm{CN}), 124.34(\mathrm{CH}), 137.04(\mathrm{CH}) ; \mathrm{MS}(\mathrm{ESI}) 147(\mathrm{M}-1)^{-}$.

Dialkylated malononitriles. These compounds were obtained in $30-75 \%$ yield from the corresponding monoalkylated malononitriles, 1,2-dibromoethane or 1,3-dibromopropane, TBAB, and potassium tert-butoxide again according to the above procedure. ${ }^{3}$

2-(2-Bromoethyl)-2-ethylmalononitrile. Yield =40\%; oil; IR $v_{\max }\left(\mathrm{cm}^{-1}\right) 2249(\mathrm{CN}) ;{ }^{1} \mathrm{H}$ NMR $(200$ MHz) $\delta 1.31(3 \mathrm{H}, \mathrm{t}, J=7.7 \mathrm{~Hz}), 2.06(2 \mathrm{H}, \mathrm{q}, J=7.7 \mathrm{~Hz}), 2.49(2 \mathrm{H}, \mathrm{br} \mathrm{t}, J=8.6 \mathrm{~Hz}), 3.59(2 \mathrm{H}$, br t, $J$ 
$=8.6 \mathrm{~Hz}) ;{ }^{13} \mathrm{C}$ NMR $(50 \mathrm{MHz}) \delta 10.38\left(\mathrm{CH}_{3}\right), 24.88\left(\mathrm{CH}_{2}\right), 32.30\left(\mathrm{CH}_{2}\right), 38.74(\mathrm{C}), 40.18\left(\mathrm{CH}_{2}\right)$, $115.01(\mathrm{CN})$; MS (EI) $m / z$ (rel inten) $202\left(\mathrm{M}^{+}+2,1\right), 200\left(\mathrm{M}^{+}, 2\right), 107(98), 79(100)$.

2-(2-Bromoethyl)-2-benzylmalononitrile. Yield $=46 \% ; \mathrm{mp}=97-98{ }^{\circ} \mathrm{C} ; \mathrm{IR} v_{\max }\left(\mathrm{cm}^{-1}\right) 2249(\mathrm{CN})$; ${ }^{1} \mathrm{H}$ NMR (300 MHz) $\delta 2.35(2 \mathrm{H}, \mathrm{t}, J=8.2 \mathrm{~Hz}), 3.11(2 \mathrm{H}, \mathrm{s}), 3.45(2 \mathrm{H}, \mathrm{t}, J=8.2 \mathrm{~Hz}), 7.22-7.38(5 \mathrm{H}$, m); ${ }^{13} \mathrm{C}$ NMR $(75 \mathrm{MHz}) \delta 20.76\left(\mathrm{CH}_{2}\right), 35.09(\mathrm{C}), 35.95\left(\mathrm{CH}_{2}\right), 39.71\left(\mathrm{CH}_{2}\right), 110.67(\mathrm{CN}), 125.49$ (CH), $125.54(\mathrm{CH}), 126.65(\mathrm{CH}), 127.70(\mathrm{C}) ; \mathrm{MS}(\mathrm{EI}) \mathrm{m} / z$ (rel inten) $264\left(\mathrm{M}^{+}+2,4\right), 262\left(\mathrm{M}^{+}, 3\right), 91$ (100); MS (ESI) $263[(\mathrm{M}+2)-1]^{-}, 261(\mathrm{M}-1)^{-}$.

2-Allyl-2-(2-bromoethyl)malononitrile. Yield = 50\%; oil; IR $v_{\max }\left(\mathrm{cm}^{-1}\right) 2249(\mathrm{CN}) ;{ }^{1} \mathrm{H}$ NMR $(200$ MHz) $\delta 2.48\left(2 \mathrm{H}, \mathrm{dd}, J_{l}=9.6 \mathrm{~Hz}, J_{2}=6.8 \mathrm{~Hz}\right), 2.74\left(2 \mathrm{H}, \mathrm{dt}, J_{d}=7.1 \mathrm{~Hz}, J_{t}=1.0 \mathrm{~Hz}\right), 3.53-3.67(2 \mathrm{H}$, m), 5.38-5.52 (2 H, m), 5.78-6.00 (1 H, m); ${ }^{13} \mathrm{C}$ NMR (50 MHz) $\delta 24.77\left(\mathrm{CH}_{2}\right), 37.46(\mathrm{C}), 39.73\left(\mathrm{CH}_{2}\right)$, $42.14\left(\mathrm{CH}_{2}\right), 114.71(\mathrm{CN}), 124.53\left(\mathrm{CH}_{2}\right), 128.39(\mathrm{CH})$; MS (ESI) $213[(\mathrm{M}+2)-1]^{-}, 211(\mathrm{M}-1)^{-}$.

2-(2-Bromoethyl)-2-[(E)-2-butenyl]malononitrile. Yield $=45 \%$; oil; IR $v_{\max }\left(\mathrm{cm}^{-1}\right) 2250(\mathrm{CN}) ;{ }^{1} \mathrm{H}$ NMR (300 MHz) $\delta 1.79(3 \mathrm{H}, \mathrm{d}, J=7.2 \mathrm{~Hz}), 2.46(2 \mathrm{H}, \mathrm{m}), 2.67(2 \mathrm{H}, \mathrm{d}, J=7.2 \mathrm{~Hz}), 3.56(2 \mathrm{H}, \mathrm{m})$, 5.44-5.60 (1 H, m), 5.77-5.92 (1 H, m); ${ }^{13} \mathrm{C} \mathrm{NMR} \mathrm{(75} \mathrm{MHz)} \delta 18.43\left(\mathrm{CH}_{3}\right), 24.96\left(\mathrm{CH}_{2}\right), 39.28\left(\mathrm{CH}_{2}\right)$, $40.94\left(\mathrm{CH}_{2}\right), 114.79(\mathrm{CN}), 120.94(\mathrm{CH}), 135.47(\mathrm{CH})$; MS (ESI) $251[(\mathrm{M}+2)+\mathrm{Na}]^{+}, 249(\mathrm{M}+\mathrm{Na})^{+}$, $229[(\mathrm{M}+2)+1]^{+}, 227(\mathrm{M}+1)^{+}$.

2-(2-Bromoethyl)-2-(3-methyl-2-butenyl)malononitrile. Yield $=41 \%$; oil; IR $v_{\max }\left(\mathrm{cm}^{-1}\right) 2249$ $(\mathrm{CN}) ;{ }^{1} \mathrm{H}$ NMR $(300 \mathrm{MHz}) \delta 1.73(3 \mathrm{H}, \mathrm{d}, J=1.1 \mathrm{~Hz}), 1.83(3 \mathrm{H}, \mathrm{d}, J=1.1 \mathrm{~Hz}), 2.44-2.49(2 \mathrm{H}, \mathrm{m})$, $2.72(2 \mathrm{H}, \mathrm{d}, J=7.7 \mathrm{~Hz}), 3.54-3.61(2 \mathrm{H}, \mathrm{m}), 5.27\left(1 \mathrm{H}, \mathrm{tq}, J_{t}=7.7 \mathrm{~Hz}, J_{q}=1.4 \mathrm{~Hz}\right) ;{ }^{13} \mathrm{C}$ NMR $(75$ MHz) $\delta 18.43\left(\mathrm{CH}_{3}\right), 24.38\left(\mathrm{CH}_{2}\right), 26.05\left(\mathrm{CH}_{3}\right), 36.44\left(\mathrm{CH}_{2}\right), 39.24\left(\mathrm{CH}_{2}\right), 113.88(\mathrm{CH}), 114.61(\mathrm{CN})$, $141.59(\mathrm{C}) ; \mathrm{MS}(\mathrm{ESI}) 241[(\mathrm{M}+2)-1]^{-}, 239(\mathrm{M}-1)^{-}, 265[(\mathrm{M}+2)+\mathrm{Na}]^{+}, 263(\mathrm{M}+\mathrm{Na})^{+}$.

2-(2-Bromoethyl)-2-[(E)-3-phenyl-2-propenyl]malononitrile. Yield $=30 \% ; \mathrm{mp}=62-64{ }^{\circ} \mathrm{C} ; \mathrm{IR} v_{\max }$ $\left(\mathrm{cm}^{-1}\right) 2250(\mathrm{CN}) ;{ }^{1} \mathrm{H} \mathrm{NMR}(200 \mathrm{MHz}) \delta 2.47(2 \mathrm{H}, \mathrm{t}, J=7.8 \mathrm{~Hz}), 2.84\left(2 \mathrm{H}, \mathrm{dd}, J_{1}=7.5 \mathrm{~Hz}, J_{2}=1.2\right.$ Hz), $3.57(2 \mathrm{H}, \mathrm{t}, J=7.8 \mathrm{~Hz}), 6.17(1 \mathrm{H}, \mathrm{m}), 6.69(1 \mathrm{H}, \mathrm{br} \mathrm{d}, J=15.5 \mathrm{~Hz}), 7.22-7.51(5 \mathrm{H}, \mathrm{m}) ;{ }^{13} \mathrm{C} \mathrm{NMR}$ $(50 \mathrm{MHz}) \delta 24.84\left(\mathrm{CH}_{2}\right), 37.85(\mathrm{C}), 39.74\left(\mathrm{CH}_{2}\right), 41.68\left(\mathrm{CH}_{2}\right), 114.83(\mathrm{CN}), 118.86(\mathrm{CH}), 127.33(\mathrm{CH})$, 
129.17 (CH), $136.00(\mathrm{C}), 138.98(\mathrm{CH})$; MS (EI) $m / z$ (rel inten) $290\left(\mathrm{M}^{+}+2,6\right), 288\left(\mathrm{M}^{+}, 8\right), 117(100)$, $91(15)$.

2-(3-Bromopropyl)-2-ethylmalononitrile. Yield = 30\%; oil; IR $v_{\max }\left(\mathrm{cm}^{-1}\right) 2241(\mathrm{CN}) ;{ }^{1} \mathrm{H}$ NMR $(200 \mathrm{MHz}) \delta 1.30(3 \mathrm{H}, \mathrm{t}, J=7.5 \mathrm{~Hz}), 1.96-2.34(6 \mathrm{H}, \mathrm{m}), 3.50(2 \mathrm{H}, \mathrm{t}, J=6.0 \mathrm{~Hz}) ;{ }^{13} \mathrm{C} \mathrm{NMR}(50 \mathrm{MHz})$ $\delta 10.51\left(\mathrm{CH}_{3}\right), 29.05\left(\mathrm{CH}_{2}\right), 31.92\left(\mathrm{CH}_{2}\right), 32.24\left(\mathrm{CH}_{2}\right), 36.65\left(\mathrm{CH}_{2}\right), 38.71(\mathrm{C}), 115.80(\mathrm{CN})$; MS (EI) $m / z$ (rel inten) $216\left(\mathrm{M}^{+}+2,2\right), 214\left(\mathrm{M}^{+}, 2\right), 186(59), 107(100)$.

2-(3-Bromopropyl)-2-[(E)-2-butenyl]malononitrile. Yield =74\%; oil; IR $v_{\max }\left(\mathrm{cm}^{-1}\right) 2249(\mathrm{CN}) ;{ }^{1} \mathrm{H}$ NMR (200 MHz) $\delta 1.79(3 \mathrm{H}$, br d, $J=6.9 \mathrm{~Hz}), 2.32-2.04(4 \mathrm{H}, \mathrm{m}), 2.65(2 \mathrm{H}, \mathrm{d}, J=7.7 \mathrm{~Hz}), 3.49(2 \mathrm{H}$, $\mathrm{t}, J=6.0 \mathrm{~Hz}), 5.43-5.62(1 \mathrm{H}, \mathrm{m}), 5.75-6.04(1 \mathrm{H}, \mathrm{m}) ;{ }^{13} \mathrm{C} \mathrm{NMR}(50 \mathrm{MHz}) \delta 14.01\left(\mathrm{CH}_{3}\right), 18.71(\mathrm{CH})$, $29.00\left(\mathrm{CH}_{2}\right), 31.89\left(\mathrm{CH}_{2}\right), 32.21\left(\mathrm{CH}_{2}\right), 36.21\left(\mathrm{CH}_{2}\right), 30.02(\mathrm{C}), 41.35\left(\mathrm{CH}_{2}\right), 115.75(\mathrm{CN}), 121.45$ (CH), $135.48(\mathrm{CH})$; MS (EI) $m / z$ (rel inten) $242\left(\mathrm{M}^{+}+2,<1\right), 240\left(\mathrm{M}^{+},<1\right), 161(10), 55(100)$.

2-(3-Bromopropyl)-2-(3-methyl-2-butenyl)malononitrile. Yield $=75 \%$; oil; IR $v_{\max }\left(\mathrm{cm}^{-1}\right) 2248$ $(\mathrm{CN}) ;{ }^{1} \mathrm{H}$ NMR $(300 \mathrm{MHz}) \delta 1.73(3 \mathrm{H}, \mathrm{d}, J=1.1 \mathrm{~Hz}), 1.82(3 \mathrm{H}, \mathrm{d}, J=0.84 \mathrm{~Hz}), 2.07-2.15(2 \mathrm{H}, \mathrm{m})$, 2.18-2.29 (2 H, m), $2.70(2 \mathrm{H}, \mathrm{d}, J=7.7 \mathrm{~Hz}), 3.49(2 \mathrm{H}, \mathrm{t}, J=6.03 \mathrm{~Hz}), 5.28\left(1 \mathrm{H}, \mathrm{t}\right.$ quint, $J_{t}=7.7 \mathrm{~Hz}$, $\left.J_{q}=1.38 \mathrm{~Hz}\right) ;{ }^{13} \mathrm{C} \mathrm{NMR}(75 \mathrm{MHz}) \delta 18.41\left(\mathrm{CH}_{3}\right), 26.03(\mathrm{CH}), 28.49\left(\mathrm{CH}_{2}\right), 31.42\left(\mathrm{CH}_{2}\right), 35.56\left(\mathrm{CH}_{2}\right)$, $36.25\left(\mathrm{CH}_{2}\right), 37.32(\mathrm{C}), 114.38(\mathrm{CH}), 115.38(\mathrm{C}), 140.91(\mathrm{CN}) ; \mathrm{MS}(\mathrm{ESI}) 279[(\mathrm{M}+2)+\mathrm{Na}]^{+}, 277$ $(\mathrm{M}+\mathrm{Na})^{+}, 257[(\mathrm{M}+2)+1)^{+}, 255(\mathrm{M}+1)^{+}$.

2-(3-Bromopropyl)-2-[(E)-3-phenyl-2-propenyl]malononitrile. Yield = 30\%; mp = 60-62 ${ }^{\circ} \mathrm{C}$; IR $v_{\max }\left(\mathrm{cm}^{-1}\right) 2251(\mathrm{CN}) ;{ }^{1} \mathrm{H}$ NMR $(200 \mathrm{MHz}) \delta 2.04-2.37(4 \mathrm{H}, \mathrm{m}), 2.85(2 \mathrm{H}, \mathrm{br} \mathrm{d}, J=8.0 \mathrm{~Hz}), 3.45(2$ $\mathrm{H}, \mathrm{t}, J=7.0 \mathrm{~Hz}), 6.20\left(1 \mathrm{H}, \mathrm{dt}, J_{d}=15.6 \mathrm{~Hz}, J_{t}=8.0 \mathrm{~Hz}\right), 6.71(1 \mathrm{H}, \mathrm{br} \mathrm{d}, J=15.6 \mathrm{~Hz}), 7.23-7.59(5 \mathrm{H}$, m); ${ }^{13} \mathrm{C}$ NMR $(50 \mathrm{MHz}) \delta 28.99\left(\mathrm{CH}_{2}\right), 31.82\left(\mathrm{CH}_{2}\right), 36.20\left(\mathrm{CH}_{2}\right), 37.87(\mathrm{C}), 41.54\left(\mathrm{CH}_{2}\right), 115.57$ $(\mathrm{CN}), 119.40(\mathrm{CH}), 127.31(\mathrm{CH}), 129.15(\mathrm{CH}), 129.32(\mathrm{CH}), 136.18(\mathrm{C}), 138.58(\mathrm{CH}) ; \mathrm{MS}(\mathrm{EI}) m / z(\mathrm{rel}$ inten) $304\left(\mathrm{M}^{+}+2,5\right), 302\left(\mathrm{M}^{+}, 6\right), 117(100), 91(13)$.

2-(2-Bromoethyl)-2-[(Z)-3-hexenyl]malononitrile. Yield = 59\%; oil; IR $v_{\max }\left(\mathrm{cm}^{-1}\right) 2249(\mathrm{CN})$; ${ }^{1} \mathrm{H}$ NMR (300 MHz) $\delta 1.00(3 \mathrm{H}, \mathrm{t}, J=7.4 \mathrm{~Hz}), 1.98-2.14(4 \mathrm{H}, \mathrm{m}), 2.35-2.53(4 \mathrm{H}, \mathrm{m}), 3.55-3.61(2 \mathrm{H}, \mathrm{m})$, 
5.26-5.35 (1 H, m), 5.50-5.58 (1 H, m); ${ }^{13} \mathrm{C}$ NMR (75 MHz) $\delta 14.65\left(\mathrm{CH}_{3}\right), 21.19\left(\mathrm{CH}_{2}\right), 23.89\left(\mathrm{CH}_{2}\right)$, $\left.24.72\left(\mathrm{CH}_{2}\right), 37.51(\mathrm{C}), 38.18\left(\mathrm{CH}_{2}\right), 40.61 \mathrm{CH}_{2}\right), 114.99(\mathrm{CN}), 124.62(\mathrm{CH}), 135.71(\mathrm{CH})$; MS (ESI) $255[(\mathrm{M}+2)-1]^{-}, 253(\mathrm{M}-1)^{-}$.

Azidated malononitriles. These compounds were obtained in 74-96\% yield from the corresponding (bromoalkyl)malononitriles and sodium azide in dimethylsulfoxide and were purified by column chromatography.

2-(2-Azidoethyl)-2-ethylmalononitrile (1a). Yield = 98\%; oil; IR $v_{\max }\left(\mathrm{cm}^{-1}\right) 2248(\mathrm{CN})$ and 2106 $\left(\mathrm{N}_{3}\right) ;{ }^{1} \mathrm{H}$ NMR $(200 \mathrm{MHz}) \delta 1.27(3 \mathrm{H}, \mathrm{t}, J=7.1 \mathrm{~Hz}), 2.04(2 \mathrm{H}, \mathrm{q}, J=7.1 \mathrm{~Hz}), 2.18(2 \mathrm{H}, \mathrm{t}, J=6.9 \mathrm{~Hz})$, $3.65(2 \mathrm{H}, \mathrm{t}, J=6.9 \mathrm{~Hz}) ;{ }^{13} \mathrm{C} \mathrm{NMR}(50 \mathrm{MHz}) \delta 9.91\left(\mathrm{CH}_{3}\right), 31.88\left(\mathrm{CH}_{2}\right), 35.93\left(\mathrm{CH}_{2}\right), 36.63(\mathrm{C}), 47.40$ $\left(\mathrm{CH}_{2}\right), 115.08(\mathrm{CN})$; MS (EI) m/z (rel inten) $135\left(\mathrm{M}^{+}-28,17\right), 106$ (34), 93 (25), 66 (49), 54 (100), 42 (99).

2-(2-Azidoethyl)-2-benzylmalononitrile (1b). Yield $=91 \% ; \mathrm{mp}=42-43{ }^{\circ} \mathrm{C} ; \mathrm{IR} \mathrm{v}_{\max }\left(\mathrm{cm}^{-1}\right) 2241$ $(\mathrm{CN})$ and $2098\left(\mathrm{~N}_{3}\right) ;{ }^{1} \mathrm{H} \mathrm{NMR}(300 \mathrm{MHz}) \delta 2.17(2 \mathrm{H}, \mathrm{t}, J=7.0 \mathrm{~Hz}), 3.24(2 \mathrm{H}, \mathrm{s}), 3.67(2 \mathrm{H}, \mathrm{t}, J=7.0$ $\mathrm{Hz}), 7.32-7.43(5 \mathrm{H}, \mathrm{m}) ;{ }^{13} \mathrm{C} \mathrm{NMR}(75 \mathrm{MHz}) \delta 36.59\left(\mathrm{CH}_{2}\right), 37.50(\mathrm{C}), 44.16\left(\mathrm{CH}_{2}\right), 47.89\left(\mathrm{CH}_{2}\right)$, $115.07(\mathrm{CN}), 129.68(\mathrm{CH}), 130.89(\mathrm{CH}), 131.99(\mathrm{C})$; MS (EI) $m / z$ (rel inten) $225\left(\mathrm{M}^{+},<1\right), 197$ (3), 118 (43), $91(100)$.

2-Allyl-2-(2-azidoethyl)malononitrile (6a). Yield =96\%; oil; IR $v_{\max }\left(\mathrm{cm}^{-1}\right) 2241$ (CN) and 2104 $\left(\mathrm{N}_{3}\right) ;{ }^{1} \mathrm{H}$ NMR $(200 \mathrm{MHz}) \delta 1.18(2 \mathrm{H}, \mathrm{t}, J=7.1 \mathrm{~Hz}), 2.75(2 \mathrm{H}, \mathrm{d}, J=7.3 \mathrm{~Hz}), 3.70(2 \mathrm{H}, \mathrm{t}, J=7.1 \mathrm{~Hz})$, 5.36-5.56 (2 H, m), 5.77-6.03 (1 H, m); ${ }^{13} \mathrm{C}$ NMR (50 MHz) $\delta 35.82(\mathrm{C}), 36.23\left(\mathrm{CH}_{2}\right), 42.44\left(\mathrm{CH}_{2}\right)$, $47.78\left(\mathrm{CH}_{2}\right), 115.00(\mathrm{CN}), 124.47\left(\mathrm{CH}_{2}\right), 128.63(\mathrm{CH})$; MS (EI) $m / z$ (rel inten) $175\left(\mathrm{M}^{+},<1\right), 119(3)$, $92(7), 39(54), 41(100)$.

2-(2-Azidoethyl)-2-[(E)-2-butenyl]malononitrile (6b). Yield = 76\%; oil; IR $v_{\max }\left(\mathrm{cm}^{-1}\right) 2254(\mathrm{CN})$ and $2107\left(\mathrm{~N}_{3}\right) ;{ }^{1} \mathrm{H}$ NMR $(300 \mathrm{MHz}) \delta 1.79(3 \mathrm{H}, \mathrm{br} \mathrm{d}, J=6.4 \mathrm{~Hz}), 2.17(2 \mathrm{H}, \mathrm{t}, J=7.0 \mathrm{~Hz}), 2.68(2 \mathrm{H}$, $\left.\mathrm{dt}, J_{d}=7.1 \mathrm{~Hz}, J_{t}=0.9 \mathrm{~Hz}\right), 3.69(2 \mathrm{H}, \mathrm{t}, J=7.0 \mathrm{~Hz}), 5.47-5.59(1 \mathrm{H}, \mathrm{m}), 5.71-6.03(1 \mathrm{H}, \mathrm{m}) ;{ }^{13} \mathrm{C} \mathrm{NMR}$ 
(75 MHz) $\delta 18.77\left(\mathrm{CH}_{3}\right), 35.96(\mathrm{C}), 36.30\left(\mathrm{CH}_{2}\right), 41.77\left(\mathrm{CH}_{2}\right), 47.92\left(\mathrm{CH}_{2}\right), 115.23(\mathrm{CN}), 121.22(\mathrm{CH})$, $135.99(\mathrm{CH})$; MS (EI) $m / z$ (rel inten) $161\left(\mathrm{M}^{+}-28,3\right), 135$ (8), 92 (11), 39 (100).

2-(2-Azidoethyl)-2-(3-methyl-2-butenyl)malononitrile (6c). Yield =90\%; oil; IR $v_{\max }\left(\mathrm{cm}^{-1}\right) 2249$ $(\mathrm{CN})$ and $2106\left(\mathrm{~N}_{3}\right) ;{ }^{1} \mathrm{H} \mathrm{NMR}(300 \mathrm{MHz}) \delta 1.73(3 \mathrm{H}$, br d, $J=1.1 \mathrm{~Hz}), 1.83(3 \mathrm{H}$, br d, $J=0.8 \mathrm{~Hz})$, $2.17(2 \mathrm{H}, \mathrm{t}, J=7.2 \mathrm{~Hz}), 2.73(2 \mathrm{H}, \mathrm{d}, J=7.7 \mathrm{~Hz}), 3.68(2 \mathrm{H}, \mathrm{t}, J=7.1 \mathrm{~Hz}), 5.28\left(1 \mathrm{H}, \mathrm{t}\right.$ quint, $J_{t}=7.7$ $\left.\mathrm{Hz}, J_{q}=1.4 \mathrm{~Hz}\right) ;{ }^{13} \mathrm{C} \mathrm{NMR}(75 \mathrm{MHz}) \delta 18.31\left(\mathrm{CH}_{3}\right), 25.91(\mathrm{CH}), 35.46\left(\mathrm{CH}_{2}\right), 36.54\left(\mathrm{CH}_{2}\right), 47.22$ $\left(\mathrm{CH}_{2}\right), 113.96(\mathrm{CH}), 114.73(\mathrm{CN}), 141.31(\mathrm{C}) ; \mathrm{MS}(\mathrm{ESI}) 226(\mathrm{M}+\mathrm{Na})^{+}$.

2-(2-Azidoethyl)-2-[(E)-3-phenyl-2-propenyl $]$ malononitrile (6d). Yield $=80 \% ; \mathrm{mp}=26-27{ }^{\circ} \mathrm{C}$; IR $v_{\max }\left(\mathrm{cm}^{-1}\right) 2249(\mathrm{CN})$ and $2094\left(\mathrm{~N}_{3}\right) ;{ }^{1} \mathrm{H}$ NMR $(200 \mathrm{MHz}) \delta 2.14(2 \mathrm{H}, \mathrm{t}, J=7.2 \mathrm{~Hz}), 2.85(2 \mathrm{H}, \mathrm{br} \mathrm{d}, J$ $=8.0 \mathrm{~Hz}), 3.64(2 \mathrm{H}, \mathrm{t}, J=7.2 \mathrm{~Hz}), 6.19\left(1 \mathrm{H}, \mathrm{dt}, J_{d}=15.2 \mathrm{~Hz}, J_{t}=8.0 \mathrm{~Hz}\right), 6.69(1 \mathrm{H}, \mathrm{br} \mathrm{d}, J=15.2$ $\mathrm{Hz}), 7.21-7.53(5 \mathrm{H}, \mathrm{m}) ;{ }^{13} \mathrm{C} \mathrm{NMR}(50 \mathrm{MHz}) \delta 36.02\left(\mathrm{CH}_{2}\right), 41.72\left(\mathrm{CH}_{2}\right), 47.68\left(\mathrm{CH}_{2}\right), 115.04(\mathrm{CN})$, $119.11(\mathrm{CH}), 127.24(\mathrm{CH}), 129.13(\mathrm{CH}), 129.26(\mathrm{CH}), 136.03(\mathrm{C}), 138.73(\mathrm{CH}) ; \mathrm{MS}(\mathrm{EI}) \mathrm{m} / z$ (rel inten) $251\left(\mathrm{M}^{+}, 2\right), 223\left(\mathrm{M}^{+}-28,18\right), 222(61), 117(100), 91(30)$.

2-(2-Azidopropyl)-2-ethylmalononitrile (1c). Yield = 85\%; oil; IR $v_{\max }\left(\mathrm{cm}^{-1}\right) 2247(\mathrm{CN})$ and 2102 $\left(\mathrm{N}_{3}\right) ;{ }^{1} \mathrm{H}$ NMR $(200 \mathrm{MHz}) \delta 1.30(3 \mathrm{H}, \mathrm{t}, J=7.5 \mathrm{~Hz}), 1.85-2.12(6 \mathrm{H}, \mathrm{m}), 3.47(2 \mathrm{H}, \mathrm{t}, J=6.0 \mathrm{~Hz}) ;{ }^{13} \mathrm{C}$ NMR $(50 \mathrm{MHz}) \delta 10.44\left(\mathrm{CH}_{3}\right), 25.89\left(\mathrm{CH}_{2}\right), 32.17\left(\mathrm{CH}_{2}\right), 35.21\left(\mathrm{CH}_{2}\right), 38.94(\mathrm{C}), 50.73\left(\mathrm{CH}_{2}\right), 115.79$ (CN); MS (EI) m/z (rel inten) $162\left(\mathrm{M}^{+}-15,2\right), 149\left(\mathrm{M}^{+}-28,5\right), 121$ (5), 107 (6), 93 (29), 79 (100), 42 (92).

2-(3-Azidopropyl)-2-[(E)-2-butenyl]malononitrile (7a). Yield =74\%; oil; IR $v_{\max }\left(\mathrm{cm}^{-1}\right) 2249$ (CN) and $2100\left(\mathrm{~N}_{3}\right) ;{ }^{1} \mathrm{H}$ NMR $(300 \mathrm{MHz}) \delta 1.78(3 \mathrm{H}, \mathrm{br} \mathrm{d}, J=6.6 \mathrm{~Hz}), 1.87-2.05(4 \mathrm{H}, \mathrm{m}), 2.59(2 \mathrm{H}, \mathrm{br} \mathrm{dd}$, $\left.J_{l}=7.4 \mathrm{~Hz}, J_{2}=0.8 \mathrm{~Hz}\right), 3.44(2 \mathrm{H}, \mathrm{m}), 5.46-5.56(1 \mathrm{H}, \mathrm{m}), 5.78-5.89(1 \mathrm{H}, \mathrm{m}) ;{ }^{13} \mathrm{C} \mathrm{NMR}(75 \mathrm{MHz}) \delta$ $13.74\left(\mathrm{CH}_{3}\right), 18.46(\mathrm{CH}), 25.64\left(\mathrm{CH}_{2}\right), 45.50\left(\mathrm{CH}_{2}\right), 38.07(\mathrm{C}), 41.03\left(\mathrm{CH}_{2}\right), 50.55\left(\mathrm{CH}_{2}\right), 115.61(\mathrm{CN})$, $121.38(\mathrm{CH}), 135.10(\mathrm{CH}) ; \mathrm{MS}(\mathrm{EI}) m / z$ (rel inten) $188\left(\mathrm{M}^{+}-15,<1\right), 55(100)$.

2-(3-Azidopropyl)-2-(3-methyl-2-butenyl)malononitrile (7b). Yield $=86 \%$; oil; IR $v_{\max }\left(\mathrm{cm}^{-1}\right)$ $2248(\mathrm{CN})$ and $2101\left(\mathrm{~N}_{3}\right) ;{ }^{1} \mathrm{H}$ NMR $(400 \mathrm{MHz}) \delta 1.73(3 \mathrm{H}, \mathrm{d}, J=0.9 \mathrm{~Hz}), 1.83(3 \mathrm{H}, \mathrm{d}, J=0.9 \mathrm{~Hz})$, 
$1.99(4 \mathrm{H}, \mathrm{m}), 2.69(2 \mathrm{H}, \mathrm{d}, J=7.7 \mathrm{~Hz}), 3.46(2 \mathrm{H}, \mathrm{t}, J=6.0 \mathrm{~Hz}), 5.28\left(1 \mathrm{H}, \mathrm{t}\right.$ quint, $J_{t}=7.7 \mathrm{~Hz}, J_{q}=$ $1.4 \mathrm{~Hz}) ;{ }^{13} \mathrm{C}$ NMR $(100 \mathrm{MHz}) \delta 19.04\left(\mathrm{CH}_{3}\right), 26.03\left(\mathrm{CH}_{2}\right), 26.67\left(\mathrm{CH}_{3}\right), 34.97\left(\mathrm{CH}_{2}\right), 37.05\left(\mathrm{CH}_{2}\right)$, $38.23(\mathrm{C}), 50.82\left(\mathrm{CH}_{2}\right), 114.96(\mathrm{CH}), 116.01(\mathrm{CN}), 141.67(\mathrm{C})$; MS (ESI) $240(\mathrm{M}+\mathrm{Na})^{+}, 218(\mathrm{M}+1)^{+}$. 2-(3-Azidopropyl)-2-[(E)-3-phenyl-2-propenyl $]$ malononitrile $(\mathbf{7 c})$. Yield $=82 \%$; $\mathrm{mp}=27-28{ }^{\circ} \mathrm{C}$; IR $v_{\max }\left(\mathrm{cm}^{-1}\right) 2249(\mathrm{CN})$ and $2097\left(\mathrm{~N}_{3}\right) ;{ }^{1} \mathrm{H}$ NMR $(200 \mathrm{MHz}) \delta 1.81-2.12(4 \mathrm{H}, \mathrm{m}), 2.83\left(2 \mathrm{H}, \mathrm{dd}, J_{l}=\right.$ $\left.8.0 \mathrm{~Hz}, J_{2}=1.2 \mathrm{~Hz}\right), 3.40(2 \mathrm{H}, \mathrm{t}, J=7.0 \mathrm{~Hz}), 6.19\left(1 \mathrm{H}, \mathrm{d} \mathrm{t}, J_{d}=15.2 \mathrm{~Hz}, J_{t}=8.0 \mathrm{~Hz}\right), 6.68(1 \mathrm{H}, \mathrm{br} \mathrm{d}, J$ $=15.2 \mathrm{~Hz}), 7.23-7.54(5 \mathrm{H}, \mathrm{m}) ;{ }^{13} \mathrm{C} \mathrm{NMR}(50 \mathrm{MHz}) \delta 25.78\left(\mathrm{CH}_{2}\right), 34.76\left(\mathrm{CH}_{2}\right), 38.06(\mathrm{C}), 41.49$ $\left(\mathrm{CH}_{2}\right), 50.57\left(\mathrm{CH}_{2}\right), 115.54(\mathrm{CN}), 119.40(\mathrm{CH}), 127.16(\mathrm{CH}), 127.25(\mathrm{CH}), 129.08(\mathrm{CH}), 129.27(\mathrm{CH})$, $136.14(\mathrm{C}), 138.44(\mathrm{CH})$; MS (EI) $m / z$ (rel inten) $265\left(\mathrm{M}^{+},<1\right), 237\left(\mathrm{M}^{+}-28,14\right) 236(43), 158(44)$ $117(81), 115$ (100), $91(71)$.

2-(2-Azidoethyl)-2-[(Z)-3-hexenyl]malononitrile (8). Yield = 80\%; oil; IR $v_{\max }\left(\mathrm{cm}^{-1}\right) 2249(\mathrm{CN})$ and $2105\left(\mathrm{~N}_{3}\right) ;{ }^{1} \mathrm{H}$ NMR $(300 \mathrm{MHz}) \delta 1.00(3 \mathrm{H}, \mathrm{t}, J=7.4 \mathrm{~Hz}), 1.92-2.15(4 \mathrm{H}, \mathrm{m}), 2.21(2 \mathrm{H}, \mathrm{t}, J=7.1$ Hz), 2.32-2.50 (2 H, m), $3.70(2 \mathrm{H}, \mathrm{t}, J=7.1 \mathrm{~Hz}), 5.26-5.36(1 \mathrm{H}, \mathrm{m}), 5.48-5.59(1 \mathrm{H}, \mathrm{m}) ;{ }^{13} \mathrm{C}$ NMR $(75$ MHz) $\delta 14.75\left(\mathrm{CH}_{3}\right), 21.30\left(\mathrm{CH}_{2}\right), 24.04\left(\mathrm{CH}_{2}\right), 35.90(\mathrm{C}), 37.24\left(\mathrm{CH}_{2}\right), 38.59\left(\mathrm{CH}_{2}\right), 47.85\left(\mathrm{CH}_{2}\right)$, $115.29(\mathrm{CN}), 124.78(\mathrm{CH}), 135.80(\mathrm{CH})$; MS (ESI) $240(\mathrm{M}+\mathrm{Na})^{+}$.

\section{General Procedure for the Reactions of Azidomalononitriles with Tributyltin Hydride. A} benzene $(15 \mathrm{~mL})$ solution of tributyltin hydride $(5.5 \mathrm{mmol})$ and AIBN $(0.5 \mathrm{mmol})$ was added by a syringe pump in $3 \mathrm{~h}$ to a refluxing benzene $(100 \mathrm{~mL})$ solution of azidomalononitrile $(5 \mathrm{mmol})$. The resulting mixture was refluxed for an additional hour. The solvent was then removed and the residue was chromatographed on silica gel; addition of methanol to the eluant was always necessary to elute the reaction product(s). In some cases $(\mathbf{6 c}, 7 \mathbf{a})$, most of the main product precipitated by concentration of the mixture and addition of a small amount of acetone; the solid was collected by filtration and the filtrate was chromatographed to obtain a further amount of product: the reported yields refer to the whole isolated quantity. In two cases $(\mathbf{1 b}, \mathbf{c})$, column chromatography was followed by a destannylation 
procedure $^{7}$ and finally by a further chromatography. All the azides were recovered completely unchanged after a 4-h reflux in benzene solution in the absence of stannane and AIBN. The yields of the main reaction products are reported in Table 1; physical and spectroscopic characterizations are described below.

3-Ethyl-2-imino-3-pyrrolidinecarbonitrile (3a). $\mathrm{mp}=93-95{ }^{\circ} \mathrm{C}$; IR $v_{\max }\left(\mathrm{cm}^{-1}\right) 3404(\mathrm{NH}), 2234$ $(\mathrm{CN})$ and $1664(\mathrm{C}=\mathrm{N}) ;{ }^{1} \mathrm{H}$ NMR $(200 \mathrm{MHz}) \delta 1.13(3 \mathrm{H}, \mathrm{t}, J=7.3 \mathrm{~Hz}), 1.62-1.81(1 \mathrm{H}, \mathrm{m}), 1.86-2.05(1$ $\mathrm{H}, \mathrm{m}), 2.15\left(1 \mathrm{H}, \mathrm{ddd}, J_{I}=13.0 \mathrm{~Hz}, J_{2}=7.2 \mathrm{~Hz}, J_{3}=4.2 \mathrm{~Hz}\right), 2.45\left(1 \mathrm{H}, \mathrm{ddd}, J_{I}=13.0 \mathrm{~Hz}, J_{2}=8.5 \mathrm{~Hz}\right.$, $\left.J_{3}=7.2 \mathrm{~Hz}\right), 3.48-3.76(2 \mathrm{H}, \mathrm{m}), 5.56(2 \mathrm{H}, \mathrm{br} \mathrm{s}, \mathrm{NH}) ;{ }^{13} \mathrm{C} \mathrm{NMR}(50 \mathrm{MHz}) \delta 10.27\left(\mathrm{CH}_{3}\right), 29.45\left(\mathrm{CH}_{2}\right)$, $35.92\left(\mathrm{CH}_{2}\right), 49.47(\mathrm{C}), 53.38\left(\mathrm{CH}_{2}\right), 121.30(\mathrm{CN}), 163.63(\mathrm{C}=\mathrm{N})$; MS (EI) $m / z$ (rel inten) $137\left(\mathrm{M}^{+}, 46\right)$, 122 (11), 108 (11), 56 (100). Anal. calcd for $\mathrm{C}_{7} \mathrm{H}_{11} \mathrm{~N}_{3}: \mathrm{C}, 61.29 ; \mathrm{H}, 8.08 ; \mathrm{N}, 30.63$. Found: $\mathrm{C}, 61.40 ; \mathrm{H}$, $8.09 ; \mathrm{N}, 30.51$.

3-Benzyl-2-imino-3-pyrrolidinecarbonitrile (3b). $\mathrm{mp}=160-161{ }^{\circ} \mathrm{C}$; IR $v_{\max }\left(\mathrm{cm}^{-1}\right) 3400(\mathrm{NH})$, $2234(\mathrm{CN})$ and $1664(\mathrm{C}=\mathrm{N}) ;{ }^{1} \mathrm{H}$ NMR $(300 \mathrm{MHz}) \delta 2.22-2.43(2 \mathrm{H}, \mathrm{m}), 3.00(1 \mathrm{H}, \mathrm{A}$ part of $\mathrm{AB}, J=$ $13.7 \mathrm{~Hz}), 3.13(1 \mathrm{H}$, B part of AB, $J=13.7 \mathrm{~Hz}), 3.28-3.37(1 \mathrm{H}, \mathrm{m}), 3.63\left(1 \mathrm{H}\right.$, ddd, $J_{1}=11.4 \mathrm{~Hz}, J_{2}=$ $\left.8.3 \mathrm{~Hz}, J_{3}=3.0 \mathrm{~Hz}\right), 4.85(2 \mathrm{H}, \mathrm{br} \mathrm{s}, \mathrm{NH}), 7.31-7.39(5 \mathrm{H}, \mathrm{m}) ;{ }^{13} \mathrm{C} \mathrm{NMR}(75 \mathrm{MHz}) \delta 36.53\left(\mathrm{CH}_{2}\right), 41.82$ $\left(\mathrm{CH}_{2}\right), 49.83(\mathrm{C}), 53.56\left(\mathrm{CH}_{2}\right), 121.35(\mathrm{CN}), 128.56(\mathrm{CH}), 129.34(\mathrm{CH}), 130.70(\mathrm{CH}), 135.00(\mathrm{C})$, $162.89(\mathrm{C}=\mathrm{N})$; MS (EI) $m / z$ (rel inten) $199\left(\mathrm{M}^{+}, 8\right), 91$ (100). Anal. caled for $\mathrm{C}_{12} \mathrm{H}_{13} \mathrm{~N}_{3}: \mathrm{C}, 72.33 ; \mathrm{H}$, 6.58; N, 21.09. Found: C, 72.28; H, 6.59; N, 21.13.

3-Ethyl-2-oxo-3-piperidinecarbonitrile (4). $\mathrm{mp}=94-96^{\circ} \mathrm{C}$; IR $v_{\max }\left(\mathrm{cm}^{-1}\right) 3396(\mathrm{NH}), 2234(\mathrm{CN})$ and $1675(\mathrm{C}=\mathrm{O}) ;{ }^{1} \mathrm{H}$ NMR $(300 \mathrm{MHz}) \delta 1.11(3 \mathrm{H}, \mathrm{t}, J=7.5 \mathrm{~Hz}), 1.80-2.30(6 \mathrm{H}, \mathrm{m}), 3.25-3.48(2 \mathrm{H}$, m), $7.12\left(1 \mathrm{H}\right.$, br s, NH); ${ }^{13} \mathrm{C}$ NMR $(75 \mathrm{MHz}) \delta 9.63\left(\mathrm{CH}_{3}\right), 19.75\left(\mathrm{CH}_{2}\right), 30.19\left(\mathrm{CH}_{2}\right), 30.70\left(\mathrm{CH}_{2}\right)$, $42.82\left(\mathrm{CH}_{2}\right), 45.01(\mathrm{C}), 121.18(\mathrm{CN}), 168.03(\mathrm{C}=\mathrm{O})$; MS (EI) $\mathrm{m} / z$ (rel inten) $152\left(\mathrm{M}^{+}, 5\right), 137(27), 124$ (100), 82 (93). Anal. calcd for $\mathrm{C}_{8} \mathrm{H}_{12} \mathrm{~N}_{2} \mathrm{O}: \mathrm{C}, 63.13 ; \mathrm{H}, 7.95 ; \mathrm{N}, 18.41$. Found: $\mathrm{C}, 63.02 ; \mathrm{H}, 7.94 ; \mathrm{N}$, 18.50 . 
1-Isoindolinimine (5). ${ }^{8} \mathrm{IR} v_{\max }\left(\mathrm{cm}^{-1}\right) 3417(\mathrm{NH}), 3326(\mathrm{NH})$ and $1637(\mathrm{C}=\mathrm{N}) ;{ }^{1} \mathrm{H}$ NMR $(200 \mathrm{MHz})$

$\delta 3.80(2 \mathrm{H}$, br s), $4.70(2 \mathrm{H}, \mathrm{s}), 7.38-8.00(3 \mathrm{H}, \mathrm{m}), 8.15(1 \mathrm{H}, \mathrm{d}, J=8.5 \mathrm{~Hz})$; MS (EI) $m / z$ (rel inten) $132\left(\mathrm{M}^{+}, 88\right), 104$ (100), 77 (54). Anal. calcd for $\mathrm{C}_{8} \mathrm{H}_{8} \mathrm{~N}_{2}$ : C, 72.70; H, 6.10; N, 21.20. Found: C, 72.77; H, 6.12; N, 21.11 .

3-Allyl-2-imino-3-pyrrolidinecarbonitrile (9a). IR $v_{\max }\left(\mathrm{cm}^{-1}\right) 3402(\mathrm{NH}), 2236(\mathrm{CN})$ and 1675 $(\mathrm{C}=\mathrm{N})$; MS (EI) $m / z$ (rel inten) $149\left(\mathrm{M}^{+}, 34\right), 108(20), 41(100)$. No reliable NMR spectra could be obtained due to product insolubility. Anal. calcd for $\mathrm{C}_{8} \mathrm{H}_{11} \mathrm{~N}_{3}: \mathrm{C}, 64.40 ; \mathrm{H}, 7.43 ; \mathrm{N}, 28.16$. Found: C, 64.53; H, 7.40; N, 28.07.

5-Ethyl-2,3,4,5-tetrahydropyrrolo[2,3-b]pyrrolo-3a(1H)-carbonitrile (10b). $\mathrm{mp}=147.5-149.4{ }^{\circ} \mathrm{C}$; IR $v_{\max }\left(\mathrm{cm}^{-1}\right) 3451(\mathrm{NH}), 2236(\mathrm{CN})$ and $1690(\mathrm{C}=\mathrm{N}) ;{ }^{1} \mathrm{H}$ NMR $(300 \mathrm{MHz}) \delta 0.98(3 \mathrm{H}, \mathrm{t}, J=7.4 \mathrm{~Hz})$, 1.45-1.77 $(3 \mathrm{H}, \mathrm{m}), 1.98\left(1 \mathrm{H}, \mathrm{ddd}, J_{l}=12.4 \mathrm{~Hz}, J_{2}=10.4 \mathrm{~Hz}, J_{3}=8.0 \mathrm{~Hz}\right), 2.46\left(1 \mathrm{H}, \mathrm{dd}, J_{1}=12.4 \mathrm{~Hz}\right.$, $\left.J_{2}=4.8 \mathrm{~Hz}\right), 2.56\left(1 \mathrm{H}, \mathrm{dd}, J_{1}=12.4 \mathrm{~Hz}, J_{2}=4.7 \mathrm{~Hz}\right), 3.83\left(1 \mathrm{H}, \mathrm{dd}, J_{1}=11.8 \mathrm{~Hz}, J_{2}=7.9 \mathrm{~Hz}\right), 4.01(1$ $\left.\mathrm{H}, \mathrm{td}, J_{t}=10.9 \mathrm{~Hz}, J_{d}=5.0 \mathrm{~Hz}\right), 4.24(1 \mathrm{H}, \mathrm{m}), 5.76(1 \mathrm{H}$, br s, NH$) ;{ }^{13} \mathrm{C} \mathrm{NMR}(75 \mathrm{MHz}) \delta 10.64$ $\left(\mathrm{CH}_{3}\right), 29.11\left(\mathrm{CH}_{2}\right), 36.41\left(\mathrm{CH}_{2}\right), 42.38\left(\mathrm{CH}_{2}\right), 49.54(\mathrm{C}), 54.90\left(\mathrm{CH}_{2}\right), 69.79(\mathrm{CH}), 119.42(\mathrm{CN})$, $170.17(\mathrm{C}=\mathrm{N})$; MS (EI) $m / z$ (rel inten) $163\left(\mathrm{M}^{+}, 43\right), 148(8), 134$ (100). Anal. calcd for $\mathrm{C}_{9} \mathrm{H}_{13} \mathrm{~N}_{3}: \mathrm{C}_{\text {, }}$ 66.23; H, 8.03; N, 25.74. Found: C, 66.15; H, 8.06; N, 25.79.

5-Isopropyl-2,3,4,5-tetrahydropyrrolo[2,3-b]pyrrolo-3a(1H)-carbonitrile (10c). IR $v_{\max }\left(\mathrm{cm}^{-1}\right)$ $3453(\mathrm{NH}), 2236(\mathrm{CN})$ and $1690(\mathrm{C}=\mathrm{N}) ;{ }^{1} \mathrm{H}$ NMR $(300 \mathrm{MHz}) \delta 0.90(3 \mathrm{H}, \mathrm{d}, J=6.6 \mathrm{~Hz}), 1.02(3 \mathrm{H}, \mathrm{d}$, $J=6.6 \mathrm{~Hz}), 1.56\left(1 \mathrm{H}, \mathrm{dd}, J_{l}=12.4 \mathrm{~Hz}, J_{2}=10.2 \mathrm{~Hz}\right), 1.69(1 \mathrm{H}$, sext, $J=6.6 \mathrm{~Hz}), 1.97\left(1 \mathrm{H}, \mathrm{ddd}, J_{l}=\right.$ $\left.12.4 \mathrm{~Hz}, J_{2}=10.4 \mathrm{~Hz}, J_{3}=8.0 \mathrm{~Hz}\right), 2.47\left(1 \mathrm{H}, \mathrm{dd}, J_{1}=12.4 \mathrm{~Hz}, J_{2}=5.0 \mathrm{~Hz}\right), 2.52\left(1 \mathrm{H}, \mathrm{dd}, J_{1}=12.4\right.$ $\left.\mathrm{Hz}, J_{2}=4.7 \mathrm{~Hz}\right), 3.84\left(1 \mathrm{H}, \mathrm{dd}, J_{1}=12.1 \mathrm{~Hz}, J_{2}=8.0 \mathrm{~Hz}\right), 3.95-4.09(2 \mathrm{H}, \mathrm{m}), 6.70(1 \mathrm{H}, \mathrm{br} \mathrm{s}, \mathrm{NH}) ;{ }^{13} \mathrm{C}$ NMR (100 MHz) $\delta 18.46\left(\mathrm{CH}_{3}\right), 20.01\left(\mathrm{CH}_{3}\right), 35.58(\mathrm{CH}), 36.46\left(\mathrm{CH}_{2}\right), 40.54\left(\mathrm{CH}_{2}\right), 49.51(\mathrm{C}), 55.14$ $\left(\mathrm{CH}_{2}\right), 74.45(\mathrm{CH}), 119.48(\mathrm{CN}), 170.05(\mathrm{C}=\mathrm{N})$; MS (ESI) $178(\mathrm{M}+1)^{+}, 176(\mathrm{M}-1)^{-}$. Anal. calcd for $\mathrm{C}_{10} \mathrm{H}_{15} \mathrm{~N}_{3}: \mathrm{C}, 67.76 ; \mathrm{H}, 8.53 ; \mathrm{N}, 23.71$. Found: $\mathrm{C}, 67.87 ; \mathrm{H}, 8.51 ; \mathrm{N}, 23.62$. 
5-Benzyl-2,3,4,5-tetrahydropyrrolo[2,3-b]pyrrolo-3a-(1H)-carbonitrile (10d). $\mathrm{mp}=223-225{ }^{\circ} \mathrm{C}$

(from ethanol); IR $v_{\max }\left(\mathrm{cm}^{-1}\right) 3448(\mathrm{NH}), 2236(\mathrm{CN})$ and $1689(\mathrm{C}=\mathrm{N}) ;{ }^{1} \mathrm{H}$ NMR $(300 \mathrm{MHz}) \delta 1.59(1$ $\left.\mathrm{H}, \mathrm{dd}, J_{1}=12.7 \mathrm{~Hz}, J_{2}=10.0 \mathrm{~Hz}\right), 1.87-2.03(1 \mathrm{H}, \mathrm{m}), 2.48\left(2 \mathrm{H}, \mathrm{ddd}, J_{1}=14.5 \mathrm{~Hz}, J_{2}=12.7 \mathrm{~Hz}, J_{3}=\right.$ $4.5 \mathrm{~Hz}), 2.74-2.95(2 \mathrm{H}, \mathrm{m}), 3.61\left(1 \mathrm{H}, \mathrm{dd}, J_{1}=11.8 \mathrm{~Hz}, J_{2}=8.2 \mathrm{~Hz}\right), 3.79-3.93(1 \mathrm{H}, \mathrm{m}), 4.54-4.69$ (1 H, m), 6.40 (1 H, br s, NH), 7.15-7.39 (5 H, m); ${ }^{13} \mathrm{C}$ NMR (75 MHz) $\delta 36.72\left(\mathrm{CH}_{2}\right), 43.32\left(\mathrm{CH}_{2}\right), 43.53$ $\left(\mathrm{CH}_{2}\right), 50.17(\mathrm{C}), 53.82\left(\mathrm{CH}_{2}\right), 71.43(\mathrm{CH}), 119.96(\mathrm{CN}), 127.23(\mathrm{CH}), 129.22(\mathrm{CH}), 129.28(\mathrm{CH})$, $138.86(\mathrm{C}), 170.77(\mathrm{C}=\mathrm{N})$; MS (EI) $\mathrm{m} / z$ (rel inten) $225\left(\mathrm{M}^{+}, 4\right), 134(100), 91(12)$. The structure of 10d was also confirmed by X-ray diffraction (see below). Anal. calcd for $\mathrm{C}_{14} \mathrm{H}_{15} \mathrm{~N}_{3}$ : C, 76.64; $\mathrm{H}, 6.71 ; \mathrm{N}$, 18.65. Found: C, 74.69; H, 6.74; N, 18.57 .

\section{2-Ethyl-2,3,4,5,6,7-hexahydro-3aH-pyrrolo[2,3-b]pyridino-3a-carbonitrile (11a). mp = 170-171.5} ${ }^{\circ} \mathrm{C}$; IR $v_{\max }\left(\mathrm{cm}^{-1}\right) 3451(\mathrm{NH}), 2225(\mathrm{CN})$ and $1665(\mathrm{C}=\mathrm{N}) ;{ }^{1} \mathrm{H}$ NMR $(300 \mathrm{MHz}) \delta 1.00(3 \mathrm{H}, \mathrm{t}, J=7.4$ Hz), 1.32-1.52 (2 H, m), $1.56\left(1 \mathrm{H}, \mathrm{dd}, J_{1}=13.2 \mathrm{~Hz}, J_{2}=3.3 \mathrm{~Hz}\right), 1.63-1.77(1 \mathrm{H}, \mathrm{m}), 1.85-1.94(1 \mathrm{H}$, m), 2.05-2.21 (1 H, m), $2.41\left(1 \mathrm{H}, \mathrm{dt}, J_{d}=13.2 \mathrm{~Hz}, J_{t}=3.3 \mathrm{~Hz}\right), 2.60\left(1 \mathrm{H}, \mathrm{dd}, J_{1}=12.4 \mathrm{~Hz}, J_{2}=5.0\right.$ $\mathrm{Hz}), 3.14\left(1 \mathrm{H}, \mathrm{td}, J_{t}=11.8 \mathrm{~Hz}, J_{d}=4.4 \mathrm{~Hz}\right), 3.36\left(1 \mathrm{H}, \mathrm{dd}, J_{1}=11.8 \mathrm{~Hz}, J_{2}=5.0 \mathrm{~Hz}\right), 3.68-3.77(1 \mathrm{H}$, m), $6.16\left(1 \mathrm{H}\right.$, br s, NH); ${ }^{13} \mathrm{C}$ NMR $(100 \mathrm{MHz}) \delta 11.66\left(\mathrm{CH}_{3}\right), 21.29\left(\mathrm{CH}_{2}\right), 29.92\left(\mathrm{CH}_{2}\right), 33.33\left(\mathrm{CH}_{2}\right)$, $43.38\left(\mathrm{CH}_{2}\right), 44.22\left(\mathrm{CH}_{2}\right), 44.68(\mathrm{C}), 65.53(\mathrm{CH}), 121.13(\mathrm{CN}), 161.61(\mathrm{C}=\mathrm{N})$; MS (ESI) $178(\mathrm{M}+1)^{+}$. Anal. calcd for $\mathrm{C}_{10} \mathrm{H}_{15} \mathrm{~N}_{3}$ : C, 67.76; H, 8.53; N, 23.71. Found: C, 67.74; H, 8.51; N, 23.75.

2-Isopropyl-2,3,4,5,6,7-hexahydro-3a $H$-pyrrolo[2,3-b]pyridino-3a-carbonitrile $(11 b) . \mathrm{mp}=172-$ $174{ }^{\circ} \mathrm{C}$; IR $v_{\max }\left(\mathrm{cm}^{-1}\right) 3397(\mathrm{NH}), 2235(\mathrm{CN})$ and $1653(\mathrm{C}=\mathrm{N}) ;{ }^{1} \mathrm{H}$ NMR $(400 \mathrm{MHz}) \delta 0.90(3 \mathrm{H}, \mathrm{d}, J=$ 6.7 Hz), $1.06(3 \mathrm{H}, \mathrm{d}, J=6.7 \mathrm{~Hz}), 1.47\left(1 \mathrm{H}, \mathrm{dd}, J_{1}=12.5 \mathrm{~Hz}, J_{2}=9.8 \mathrm{~Hz}\right), 1.53\left(1 \mathrm{H}, \mathrm{td}, J_{t}=13.2 \mathrm{~Hz}\right.$, $\left.J_{d}=3.3 \mathrm{~Hz}\right), 1.63(1 \mathrm{H}, \mathrm{m}), 1.85-1.94(1 \mathrm{H}, \mathrm{m}), 2.07-2.21(1 \mathrm{H}, \mathrm{m}), 2.42\left(1 \mathrm{H}, \mathrm{dt}, J_{d}=13.2 \mathrm{~Hz}, J_{t}=3.3\right.$ $\mathrm{Hz}), 2.55\left(1 \mathrm{H}, \mathrm{dd}, J_{1}=12.3 \mathrm{~Hz}, J_{2}=5.1 \mathrm{~Hz}\right), 3.15\left(1 \mathrm{H}, \mathrm{td}, J_{t}=11.8 \mathrm{~Hz}, J_{d}=4.2 \mathrm{~Hz}\right), 3.39(1 \mathrm{H}, \mathrm{dddd}$, $\left.J_{1}=11.7 \mathrm{~Hz}, J_{2}=5.5 \mathrm{~Hz}, J_{3}=2.0 \mathrm{~Hz}, J_{4}=1.1 \mathrm{~Hz}\right), 3.53\left(1 \mathrm{H}, \mathrm{ddd}, J_{1}=13.0 \mathrm{~Hz}, J_{2}=7.9 \mathrm{~Hz}, J_{3}=5.1\right.$ $\mathrm{Hz}), 5.00\left(1 \mathrm{H}\right.$, br s, NH); ${ }^{13} \mathrm{C} \mathrm{NMR}(100 \mathrm{MHz}) \delta 19.36\left(\mathrm{CH}_{3}\right), 20.96\left(\mathrm{CH}_{3}\right), 21.31\left(\mathrm{CH}_{2}\right), 33.58\left(\mathrm{CH}_{2}\right)$, $34.07(\mathrm{CH}), 42.15\left(\mathrm{CH}_{2}\right), 43.83\left(\mathrm{CH}_{2}\right), 44.59(\mathrm{C}), 70.06(\mathrm{CH}), 120.95(\mathrm{CN}), 161.41(\mathrm{C}=\mathrm{N})$; MS (ESI) 
$192(\mathrm{M}+1)^{+}$. Anal. calcd for $\mathrm{C}_{11} \mathrm{H}_{17} \mathrm{~N}_{3}: \mathrm{C}, 69.07 ; \mathrm{H}, 8.96 ; \mathrm{N}, 21.97$. Found: $\mathrm{C}, 68.95 ; \mathrm{H}, 8.99 ; \mathrm{N}$, 22.06 .

2-Benzyl-2,3,4,5,6,7-hexahydro-3aH-pyrrolo[2,3-b]pyridino-3a-carbonitrile (11c). $\mathrm{mp}=198-199$ ${ }^{\circ} \mathrm{C}$ (from ethyl acetate); IR $v_{\max }\left(\mathrm{cm}^{-1}\right) 3391(\mathrm{NH}), 2232(\mathrm{CN})$ and $1648(\mathrm{C}=\mathrm{N}) ;{ }^{1} \mathrm{H}$ NMR $(300 \mathrm{MHz}) \delta$ 1.40-1.58 (2 H, m), 1.78-1.92 (1 H, m), 1.98-2.17 (1 H, m), $2.35\left(1 \mathrm{H}, \mathrm{dt}, J_{d}=12.7 \mathrm{~Hz}, J_{t}=1.0 \mathrm{~Hz}\right), 2.46$ $\left(1 \mathrm{H}, \mathrm{dd}, J_{1}=12.7 \mathrm{~Hz}, J_{2}=5.1 \mathrm{~Hz}\right), 2.64\left(1 \mathrm{H}, \mathrm{dd}, J_{1}=14.0 \mathrm{~Hz}, J_{2}=7.7 \mathrm{~Hz}\right), 2.98-3.23(3 \mathrm{H}, \mathrm{m}), 4.03-$ $4.17(1 \mathrm{H}, \mathrm{m}), 6.38\left(1 \mathrm{H}\right.$, br s, NH), 7.15-7.38 (5 H, m); ${ }^{13} \mathrm{C}$ NMR $(75 \mathrm{MHz}) \delta 21.19\left(\mathrm{CH}_{2}\right), 33.21$ $\left(\mathrm{CH}_{2}\right), 43.08\left(\mathrm{CH}_{2}\right), 43.36\left(\mathrm{CH}_{2}\right), 44.36\left(\mathrm{CH}_{2}\right), 44.64(\mathrm{C}), 65.22(\mathrm{CH}), 120.92(\mathrm{CN}), 126.95(\mathrm{CH})$, $129.07(\mathrm{CH}), 129.59(\mathrm{CH}), 139.36(\mathrm{C}), 162.00(\mathrm{C}=\mathrm{N})$; MS (EI) $m / z$ (rel inten) $148\left(\mathrm{M}^{+}-91,100\right), 120$ (13), 91 (8). The structure of 11c was also confirmed by X-ray diffraction (see below). Anal. calcd for $\mathrm{C}_{15} \mathrm{H}_{17} \mathrm{~N}_{3}: \mathrm{C}, 75.28 ; \mathrm{H}, 7.16 ; \mathrm{N}, 17.56$. Found: C, 75.38; H, 7.13; N, 17.49.

From this reaction a small amount $(<5 \%)$ of the mono-cyclized product 2-oxo-3-[(E)-3-phenyl-2propenyl]-3-piperidinecarbonitrile was also obtained; $\mathrm{mp}=113-115^{\circ} \mathrm{C}$; IR $v_{\max }\left(\mathrm{cm}^{-1}\right) 3401(\mathrm{NH})$, $2233(\mathrm{CN})$ and $1679(\mathrm{C}=\mathrm{O}) ;{ }^{1} \mathrm{H}$ NMR $(300 \mathrm{MHz}) \delta$ 1.91-2.08 (3 H, m), 2.17-2.27 (1 H, m), 2.78-2.85 (1 H, m), 3.00-3.07 (1 H, m), 3.29-3.48 (2 H, m), $6.19\left(1 \mathrm{H}, \mathrm{dt}, J_{d}=15.1 \mathrm{~Hz}, J_{t}=7.5 \mathrm{~Hz}\right), 6.57(1 \mathrm{H}, \mathrm{br} \mathrm{d}, J$ $=15.1 \mathrm{~Hz}), 6.68\left(1 \mathrm{H}\right.$, br s, NH), 7.25-7.40 (5 H, m); ${ }^{13} \mathrm{C} \mathrm{NMR}(75 \mathrm{MHz}) \delta 19.85\left(\mathrm{CH}_{2}\right), 30.38(\mathrm{C})$, $31.04\left(\mathrm{CH}_{2}\right), 40.41\left(\mathrm{CH}_{2}\right), 43.00\left(\mathrm{CH}_{2}\right), 120.94(\mathrm{CN}), 122.85(\mathrm{CH}), 127.09(\mathrm{CH}), 128.58(\mathrm{CH}), 129.29$ (CH), $136.47(\mathrm{CH}), 137.10(\mathrm{C}), 167.13(\mathrm{CO})$; MS (EI) m/z (rel inten) $240\left(\mathrm{M}^{+}, 31\right), 117$ (100), 91 (35). Anal. calcd for $\mathrm{C}_{15} \mathrm{H}_{16} \mathrm{~N}_{2} \mathrm{O}$ : C, 74.97; H, 6.71; N, 11.66. Found: C, 74.99; H, 6.70; N, 11.63.

3-[(Z)-3-Hexenyl]-2-imino-3-pyrrolidinecarbonitrile (16). IR $v_{\max }\left(\mathrm{cm}^{-1}\right) 3406(\mathrm{NH}), 2237$ (CN) and $1666(\mathrm{C}=\mathrm{N}) ;{ }^{1} \mathrm{H}$ NMR $(400 \mathrm{MHz}) \delta 0.98(3 \mathrm{H}, \mathrm{t}, J=7.5 \mathrm{~Hz}), 1.63-1.76(1 \mathrm{H}, \mathrm{m}), 1.80-1.96(1 \mathrm{H}$, m), 1.98-2.13 (3 H, m), 2.14-2.26 (2 H, m), 2.45-2.53 (1 H, m), 3.55-3.65 (1 H, m), $3.72\left(1 \mathrm{H}, \mathrm{ddd}, J_{1}=\right.$ 8.4 Hz, $\left.J_{2}=4.0 \mathrm{~Hz}, J_{3}=1.4 \mathrm{~Hz}\right), 5.26-5.34(1 \mathrm{H}, \mathrm{m}), 5.40-5.50(1 \mathrm{H}, \mathrm{m}) 4.54-5.01(2 \mathrm{H}, \mathrm{br} \mathrm{s}, \mathrm{NH}) ;{ }^{13} \mathrm{C}$ NMR (100 MHz) $\delta 14.86\left(\mathrm{CH}_{3}\right), 21.25\left(\mathrm{CH}_{2}\right), 24.00\left(\mathrm{CH}_{2}\right), 36.21\left(\mathrm{CH}_{2}\right), 36.75\left(\mathrm{CH}_{2}\right), 48.79(\mathrm{C}), 53.88$ 
$\left(\mathrm{CH}_{2}\right), 121.19(\mathrm{CN}), 126.6(\mathrm{CH}), 134.58(\mathrm{CH}), 163.00(\mathrm{C}=\mathrm{N})$; MS (ESI) $192(\mathrm{M}+1)^{+}$. Anal. calcd for $\mathrm{C}_{11} \mathrm{H}_{17} \mathrm{~N}_{3}$ : C, 69.07; H, 8.96; N, 21.97. Found: C, 69.04; H, 8.97; N, 21.99.

From this reaction a small amount $(<5 \%)$ of 5-((E)-2-\{5-cyano-5- $[(\boldsymbol{Z})-3-h e x e n y l]-2-$ pyrrolidinylidene\}hydrazone)-2-[(Z)-3-hexenyl]-2-pyrrolidinecarbonitrile (the probable dimer of compound 16) was also obtained; MS (ESI) $403(\mathrm{M}+\mathrm{Na})^{+}, 381(\mathrm{M}+1)^{+}$.

2-Imino-3-(3-methyl-2-butenyl)-3-pyrrolidinecarbonitrile (9c). This compound was obtained by reacting azide $6 \mathbf{c}$ with tributyltin hydride in the absence of $A I B N ; m p=110.9-112.6{ }^{\circ} \mathrm{C} ; \operatorname{IR~} v_{\max }\left(\mathrm{cm}^{-1}\right)$ $3405(\mathrm{NH}), 3501(\mathrm{NH}), 2238(\mathrm{CN})$ and $1664(\mathrm{C}=\mathrm{N}) ;{ }^{1} \mathrm{H}$ NMR $(300 \mathrm{MHz}) \delta 1.68(3 \mathrm{H}, \mathrm{d}, J=1.1 \mathrm{~Hz})$, $1.78(3 \mathrm{H}, \mathrm{d}, J=0.8 \mathrm{~Hz}), 2.11-2.19\left(1 \mathrm{H}, \mathrm{ddd}, J_{1}=13.0 \mathrm{~Hz}, J_{2}=7.2 \mathrm{~Hz}, J_{3}=3.9 \mathrm{~Hz}\right), 2.34-2.50(2 \mathrm{H}$, $\left.\mathrm{dd}, J_{1}=8.5 \mathrm{~Hz}, J_{2}=7.1 \mathrm{~Hz}\right), 2.58\left(1 \mathrm{H}, \mathrm{dd}, J_{l}=14.6 \mathrm{~Hz}, J_{2}=7.7 \mathrm{~Hz}\right), 3.69\left(1 \mathrm{H}, \mathrm{ddd}, J_{l}=13.6 \mathrm{~Hz}, J_{2}\right.$ $\left.=8.5 \mathrm{~Hz}, J_{3}=3.9 \mathrm{~Hz}\right), 3.50-3.63(1 \mathrm{H}, \mathrm{m}), 5.24\left(1 \mathrm{H}, \mathrm{t}\right.$ quint, $\left.J_{t}=7.4 \mathrm{~Hz}, J_{q}=1.4 \mathrm{~Hz}\right), 4.91(2 \mathrm{H}$, br s, $\mathrm{NH}) ;{ }^{13} \mathrm{C}$ NMR $(75 \mathrm{MHz}) \delta 18.11\left(\mathrm{CH}_{3}\right), 25.91\left(\mathrm{CH}_{3}\right), 34.11\left(\mathrm{CH}_{2}\right), 35.82\left(\mathrm{CH}_{2}\right), 48.00(\mathrm{C}), 52.93$ $\left(\mathrm{CH}_{2}\right), 116.8(\mathrm{CH}), 55.14\left(\mathrm{CH}_{2}\right), 120.71(\mathrm{CN}), 138.08(\mathrm{C}), 162.60(\mathrm{C}=\mathrm{N})$; MS (ESI) $178(\mathrm{M}+1)^{+}$. Anal. calcd for $\mathrm{C}_{10} \mathrm{H}_{15} \mathrm{~N}_{3}$ : C, 67.76; H, 8.53; N, 23.71. Found: C, 67.87; H, 8.51; N, 23.62. This compound proved to be perfectly stable when kept for several hours in a refluxing benzene solution in the presence of AIBN.

2-Imino-3-[(E)-3-phenyl-2-propenyl)-3-pyrrolidinecarbonitrile (9d). This compound was obtained by reacting azide 6d with tributyltin hydride in the absence of AIBN; IR $v_{\max }\left(\mathrm{cm}^{-1}\right) 3404(\mathrm{NH}), 2238$ $(\mathrm{CN})$ and $1665(\mathrm{C}=\mathrm{N}) ;{ }^{1} \mathrm{H}$ NMR $(300 \mathrm{MHz}) \delta$ 2.18-2.32 (1 H, m), 2.39-2.54 (1 H, m), 2.55-2.69 (1 H, m), 2.70-2.82 (1 H, m), 3.50-3.77 (2 H, m), $4.78(2 \mathrm{H}$, br s, NH), $6.20(1 \mathrm{H}, \mathrm{m}), 6.60(1 \mathrm{H}, \mathrm{br} \mathrm{d}, J=16.0$ Hz), 7.16-7.48 (5 H, m); MS (EI) m/z (rel inten) $225\left(\mathrm{M}^{+}, 12\right), 117$ (100), 91 (51). Anal. calcd for $\mathrm{C}_{14} \mathrm{H}_{15} \mathrm{~N}_{3}: \mathrm{C}, 74.64 ; \mathrm{H}, 6.71 ; \mathrm{N}, 18.65$. Found: $\mathrm{C}, 74.59 ; \mathrm{H}, 6.72 ; \mathrm{N}, 18.69$. This compound proved to be perfectly stable when kept for several hours in a refluxing benzene solution in the presence of AIBN. 


\section{Single crystal X-ray analyses}

Crystallographic data were collected at $298 \mathrm{~K}$ on a Enraf-Nonius CAD4 diffractometer using Cu-Ko radiation $(\lambda=1.54178 \AA)$. Data were corrected for Lorentz and polarization effects but not for absorption. The structures were solved by direct methods (SIR97 ${ }^{9}$ ) and anisotropically refined for all the non-H atoms. The refinements were performed on $F^{2}$ using SHELX93. ${ }^{10}$ The hydrogen atoms were located from a difference Fourier map and isotropically refined with displacement parameter 1.2 greater than the $U_{\text {eq }}$ of the attached atoms. The $\mathrm{H}$ atoms attached to the carbon atoms were refined using a riding model. In 10d one hydrogen atom was found to be statistically distributed over two positions associated to the nitrogen atoms of the five-membered rings. During the refinement the site occupation factors were allowed to vary freely, converging to $0.65(3)$ and $0.35(3)$ for the positions associated to N1 and N2 respectively.

10d: $\mathrm{C}_{14} \mathrm{H}_{15} \mathrm{~N}_{3}, M_{\mathrm{r}}=225.3$, orthorhombic, space group Pbca, $a=10.819(2), b=9.869(2), c=22.487(5)$ $\AA, V=2401.0(8) \AA^{3}, Z=8, \rho_{\text {calc }}=1.246 \mathrm{~g} \mathrm{~cm}^{-3}, \mu=0.596 \mathrm{~mm}^{-1}$; colorless prism, crystal dimensions $0.18 \times 0.20 \times 0.28 \mathrm{~mm}, 161$ parameters, $R=0.047, w R 2=0.091$ (for 1809 unique reflections, $I>0$ ), $S=$ $0.938, \Delta \rho(\min / \max )=-0.12 / 0.20$ e $\AA^{-3}$

11c: $\mathrm{C}_{15} \mathrm{H}_{17} \mathrm{~N}_{3}, M_{\mathrm{r}}=239.3$, monoclinic, space group $P 2_{1} / n, a=7.504(2), b=15.354(4), c=11.812(4)$ $\AA, \beta=105.48(3)^{\circ}, V=1311.6(7) \AA^{3}, Z=4, \rho_{\text {calc }}=1.212 \mathrm{~g} \mathrm{~cm}^{-3}, \mu=0.573 \mathrm{~mm}^{-1}$; colorless prism, crystal dimensions $0.08 \times 0.13 \times 0.32 \mathrm{~mm}, 166$ parameters, $R=0.042, w R 2=0.097$ (for 2203 unique reflections, $I>0), S=0.991, \Delta \rho(\min / \max )=-0.17 / 0.03$ e $\AA^{-3}$. 


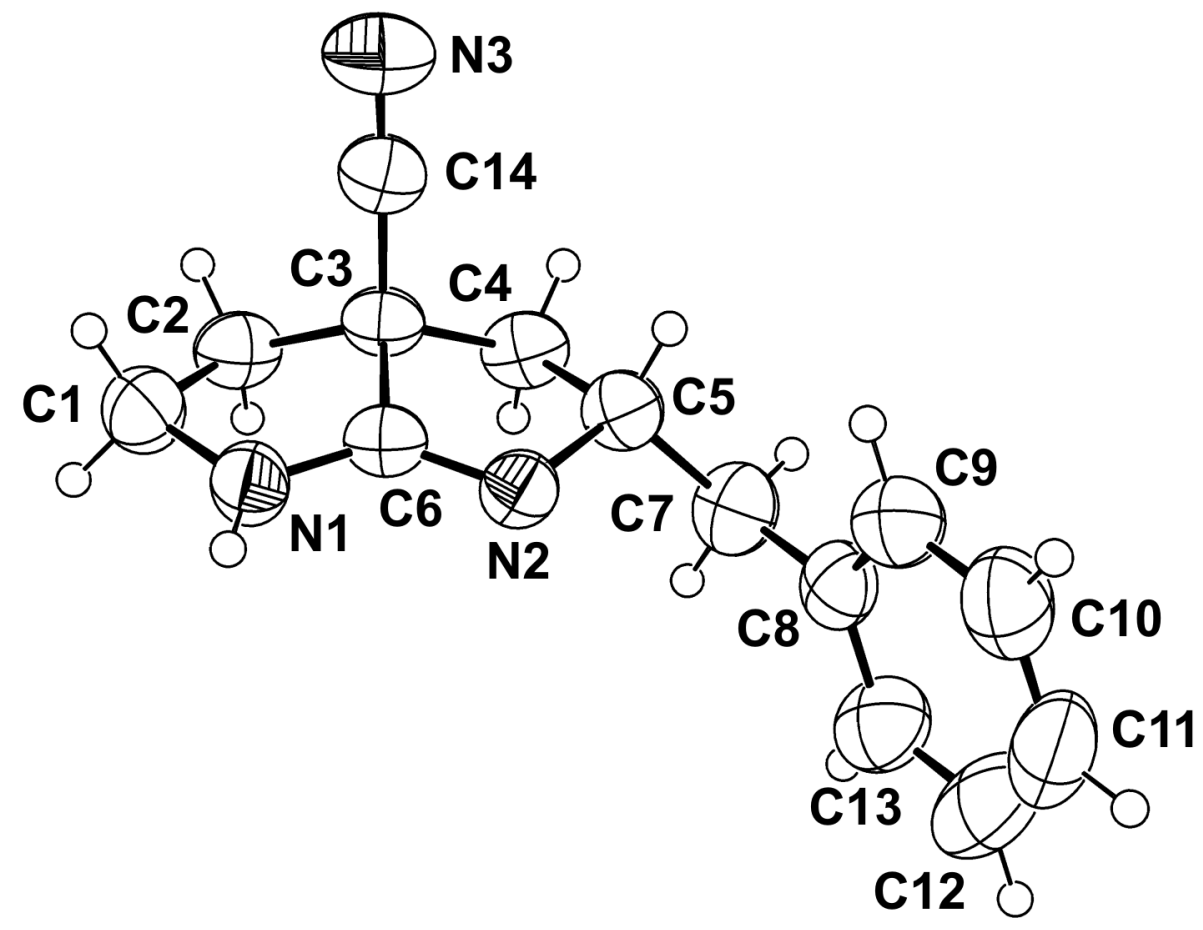

Figure 1. Single crystal X-ray structure of $\mathbf{1 0 d}$ showing the labeling scheme adopted. Only one position for the disordered hydrogen atom associated to the nitrogen atoms of the five-membered rings is given.

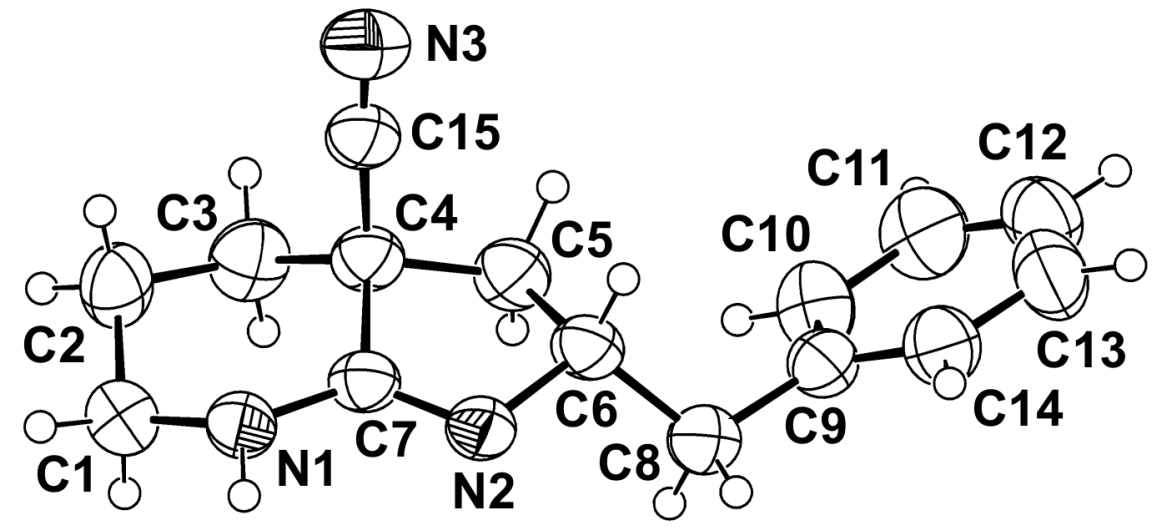

Figure 2. Single crystal X-ray structure of $11 \mathbf{c}$ showing the labeling scheme adopted. 


\section{Comment to the structures}

\section{Compound 10d.}

Both the five-membered rings assume a twist conformation.

Bond distances and angles are as expected. The values of the bond distances indicate the presence of

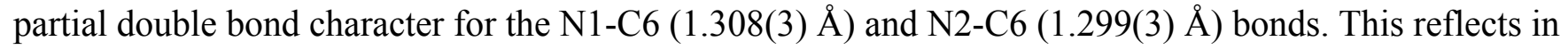
the disorder observed for the associated hydrogen atom, which was found to be statistically attached to both nitrogen atoms with site occupation factors of $0.65(3)$ and $0.35(3)$ for the position related to N1 and $\mathrm{N} 2$, respectively.

As observed in 11c (see below), in the packing centrosymmetric pairs of molecules interact through hydrogen bonds giving rise to dimers:

N1-H1N, $0.78 \AA ; \quad H 1 N \ldots . . N 2 ', 2,20 \AA ; \quad$ N1-H1N...N2', 161.4

N2-H2N, $0.76 \AA ̊ \quad H 2 N \ldots N 1 ', 2,25 \AA ; \quad \mathrm{N} 1-\mathrm{H} 1 \mathrm{~N} \ldots \mathrm{N} 22^{\prime}, 154.2^{\circ}$

(a prime denotes a transformation of $-\mathrm{x},-\mathrm{y},-\mathrm{z}$ )

\section{Compound 11c.}

The six- and five-membered rings assume a chair and twist conformation, respectively.

Bond distances and angles are as expected; the value of the N2-C7 bond distance $(1.278(2) \AA)$ is consistent with a localized double-bond character.

In the packing centrosymmetric pairs of molecules interact through hydrogen bonds resulting in the formation of dimers:

N1-H1N, $0.87 \AA ; \quad H 1 N \ldots N 2 ', 2,12 \AA ; \quad$ N1-H1N...N2', $175.4^{\circ}$

(a prime denotes a transformation of $-\mathrm{x}, 1-\mathrm{y},-\mathrm{z}$ ) 
Beckwith-Houk transition state models for cyclization of aminoiminyl $\mathbf{1 2 d}\left(\mathrm{SnBu}_{3}=\mathrm{Me}\right)$ :

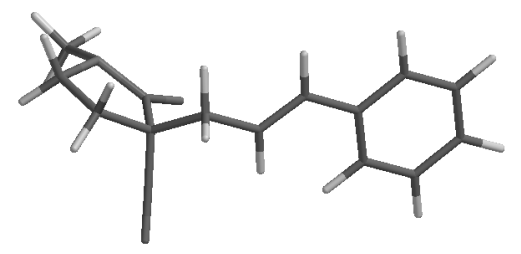

Chair-like TS (axial CN) ( $\rightarrow$ trans-bicyclic radical), favored

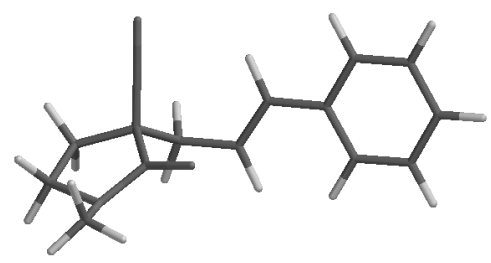

Boat-like TS ( $\rightarrow$ cis-bicyclic radical), disfavored

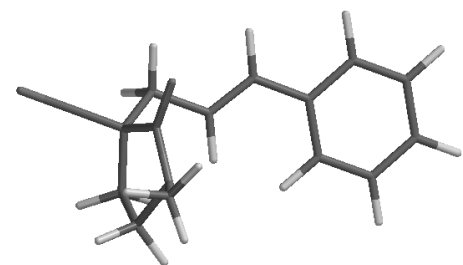

Chair-like TS (equatorial $\mathrm{CN}$ ), disfavored, the iminyl moiety is very distant from the $\mathrm{C}=\mathrm{C}$ double bond 
Optimized (DFT) structures of radicals cis-14b and trans-14b $\left(\mathrm{SnBu}_{3}=\mathrm{SnMe}_{3}\right)$, respectively:

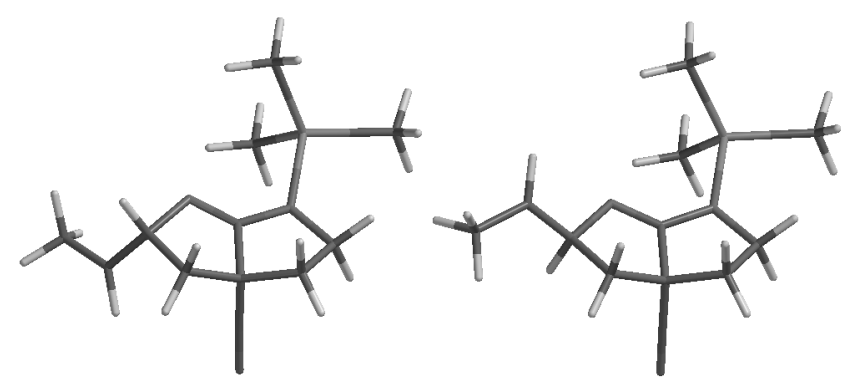

Structures were optimized with the DFT program included in the Wavefunction PC Spartan Pro 1.0.3 package. For sake of semplicity, the tributyltin group was replaced with a trimethyltin moiety. After a careful conformational search, the geometries of the open-shell intermediates were fully optimized following the $\mathrm{pBP} / \mathrm{DN}^{* *}$ parameterization. The energy minimum of cis-14b was located at -6659.75571 $\mathrm{H}$ and that of trans-14b at $-6659.75747 \mathrm{H}$, corresponding to an energy difference of $1.1 \mathrm{kcal} / \mathrm{mol}$ in favor of the trans-radical. Both structures gave $<S>^{2}$ values of 0.752 . 
Optimized (DFT) structures of 3-ethyl-2-imino-3-pyrrolidinecarbonitrile (3a), and tautomeric 5-amino4-ethyl-3,4-dihydro-2H-pyrrole-4-carbonitrile, respectively:
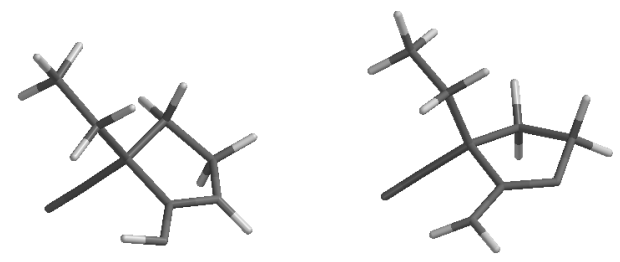

Structures were optimized with the DFT program $\left(\mathrm{pBP} / \mathrm{DN}^{* *}\right.$ parameterization) included in the Wavefunction PC Spartan Pro 1.0.3 package. The energy minimum of 3a (left structure) was located at $-437.76931 \mathrm{H}$ and that of its tautomeric amine (right structure) at $-437.77251 \mathrm{H}$, corresponding to an energy difference of $2.0 \mathrm{kcal} / \mathrm{mol}$ in favor of the latter. 


\section{References}

${ }^{1}$ Fuson, R. C. J. Am. Chem. Soc. 1926, 48, 830.

${ }^{2}$ L'Abbé, G.; Sannen, I.; Dehaen, W. J. Chem. Soc., Perkin Trans. 1 1993, 27.

${ }^{3}$ Diez-Barra, E.; De La Hoz, A.; Moreno, A.; Sanchez-Verdù, P. J. Chem. Soc., Perkin Trans. 1 1991, 2589.

${ }^{4}$ Curran, D. P.; Seong, C. M. Tetrahedron 1992, 48, 2175.

${ }^{5}$ Kolsaker, P.; Storesund, H. J.; Gulbrandsen, T.; Wøien, G. Acta Chem. Scand. 1983, B 37, 187.

${ }^{6}$ Giambastiani, G.; Poli, G. J. Org. Chem. 1998, 63, 9608.

${ }^{7}$ Kizil, M.; Patro, B.; Callaghan, O.; Murphy, J. A.; Hursthouse, M. B.; Hibbs, D. J. Org. Chem. 1999, 64, 7856 .

${ }^{8}$ (a) Sawanishi, H.; Sashida, H.; Tsuchiya, T. Chem. Pharm. Bull. 1985, 33, 4564. (b) Sieveking, H. U.; Luettke, W. Angew. Chem. Int. Ed. 1969, 8, 457.

9 Altomare, A.; Burla, M. C.; Camalli, M.; Cascarano, G. L.; Giacovazzo, C.; Guagliardi, A.; Moliterni, A. G. G.; Polidori, G.; Spagna, R. J. Appl. Cryst., 1999, 32, 115-119.

${ }^{10}$ Sheldrick, G. M. SHELXL93. Program for crystal structure refinement. University of Göttingen, Germany, 1993. 